\title{
Magnetic structure of sunspot under the photosphere
}

\author{
Elena A. Kirichek ${ }^{1}$ and Alexandr A. Solov'ev ${ }^{1}$ \\ ${ }^{1}$ Central (Pulkovo) Observatory, Russian Academy of Sciences, \\ St. Petersburg, 196140 Russia \\ email: solov@gao.spb.ru
}

In recent years, the local helioseismology has become a highly effective tool for investigating subphotospheric layers of the Sun, which can yield fairly detailed distributions of the subphotospheric temperatures and large-scale plasma flows based on the spectra of the oscillations observed at the photospheric layers and the observed peculiarities of propagation of magnetoacoustic waves in this medium (Zhao et al. (2001), Kosovichev (2006)). Unfortunately, the effects of temperature and the magnetic field on the wave propagation speed have not yet been separated Kosovichev (2006), so that the structure of the sunspot magnetic field in deep layers, beneath the photosphere, remains a subject of purely theoretical analysis. In his analysis of some theoretical models of the subphotospheric layers of sunspots based on recent helioseismological data, Kosovichev (2006) concluded that Parker's ("spaghetti") cluster model Parker (1979) is most appropriate. In this model, the magnetic flux in the sunspot umbra is concentrated into separate, strongly compressed, vertical magnetic flux tubes that are interspaced with plasma that is almost free of magnetic field; the plasma can move between these tubes.

Our study is dedicated to the problem of the magnetic field structure in the subphotospheric layers of a sunspot regarded as a long-lived (actually steady-state) well-structured stable formation. We will demonstrate that, in the cool part of the magnetic flux tube of sunspot the Parker's model can be successfully applied, but in the region of "overheating" located, in according with helioseismological data, under the cool column of the spot, at depths from about $4 \mathrm{Mm}$, the condition of transverse (horizontal) equilibrium, i.e. the lateral balance of the total (gaseous + magnetic) pressures, cannot be satisfied in Parker's sunspot model. The magnetic flux tube of the spot must expand sharply with the depth starting from depths of about $4 \mathrm{Mm}$, so that it penetrates into deeper hot layers of the convection zone in an very expanded, diffuse form, as is assumed in the "shallow sunspot" model of Efremov et al. (2007), Solovev \& Kirichek (2008).

We use this model to give the theoretical interpretation of the low-frequency eigen oscillations of sunspots observed both by ground-based telescopes (Pulkovo) and by space instruments (MDI/SOHO) Efremov et al. (2009).

\section{References}

Efremov, V. I., Parfinenko, L. D., \& Solovev, A. A. 2007, Astronomy Reports, 51, N5, 401-410. Efremov, V. I., Parfinenko, L. D., \& Solovev., A. A. 2009, (accepted). Solar Phys.

Kosovichev, A. G. 2006, Adv. Space Res., 38, 876

Parker, E. N. 1979, Cosmical Magnetic Fields, Part 1. (Claredon Press, Oxford), 608.

Solovev A. A. \& Kirichek E. A. 2008, Astrophysical Bulletin, 63, N2, 169-180

Zhao, J., Kosovichev A. G. and Duval, T. L. 2001, Astrophys. J., 557, 384 